\title{
Implementation of Virtual Reality in Serious Gaming - War Simulation
}

\author{
S. Ramachandran,s. Rabiyathul basariya
}

\begin{abstract}
In this work, the researcher presents War Simulation through virtual reality. Through the work, the researcher tries to create a tactical war kind off a simulation that will not only be beneficial to train the military personnel but also combine with the Virtual Reality in order to give an experience that will help the Indian personnel to be trained in a better way. The researcher takes into account the simulation process that is being used throughout the world and follows the steps of various already constructed simulations with added advantages and upgrades. This work shall discuss the technical aspects of the construction of the simulation while also taking into account the amount that will be used in building the simulation. By combining the elements of a serious game and combine them with Virtual reality, the simulation provides a very immersive experience that will be used to help the soldiers in a more virtual way. While starting to create the simulation the researcher takes into account the various necessities that will be used to create the simulation. There will be different multiplayer aspects that will be used in this simulation. A detailed explanation will be provided for various topics.
\end{abstract}

Keywords: Virtual Reality (VR), Photon Unity Network (PUN), Remote Procedure Calls (RPCs)

\section{INTRODUCTION}

We are trying to create a multiplayer virtual reality-based army training simulation that will help the army personnel to deal with different tactical situations. We are establishing an offline and online system. In the offline system will work for the users that require training for a single person alone. They will be able to work on different tactical situations in order to train themselves and see how they fare in these situations. In online mode, there will be a group of users that can be dynamically connected over a network and can work together. By using the online simulation, the control is over the main commander alone they will be able to use different situations and work on them. For example, they can work on how each individual can be made to work as a team. Since this is completely a virtual world it saves a lot of resources. Resources which may include excessive use of ammo and dealing with different sectors and also saving a lot of time in using different tactical terrains. As of the current situation, the multiplayer that we will be established will be set to a minimum of 4 users. In order to create this simulation, it will require a tremendous amount of resources. My team will be creating the base of the simulation program by creating a multiplayer system along with a single player training system along with which you can choose the guns and the characters

Revised Manuscript Received on December 22, 2018.

S. Ramachandran, Research Scholar, Bharath Institute of Higher Education and Research, Chennai.Email: sramchand56@ gmail.com

S. RabiyathulBasariya,Associate Professor\& Research Supervisor, Dept. of Commerce \& Business Administration, Bharath Institute of Higher Education and Research, Chennai. Email: rabiya_s08@yahoo.com. that will be used in order to play this game. There will be different map systems that we will be working so that the various army personnel will be able to use different terrains and help themselves with the knowledge on how they can use the provided terrains in their advantage. In the single-player offline situations, there will be only one person playing this game and it is completely offline meaning that they can play this game without any use of the internet connection. In this offline mode, there will be different situations that will be already made active so that the user can test and improve their skills. They can look back and see how they fare in each situation thus providing them with the results so that they can check their performance and improve them. There will be the main menu system where they will be able to navigate through the online and the offline mode. There will also be features where they can change their look and also use different guns and various other customizations. They can also change the settings according to their comfort so that they won't be at a discomfort while playing this game. Thus provided with a detailed description of how the wartime simulation will proceed. This work will require a huge amount of resources that is the reason why my team and I will be working on how to set up a base with different features and use this base to expand further so that the project doesn't get stuck and closes abruptly[10-16].

\section{EXISTING SySTEM}

The commercialization exertion of Virtual Reality innovation has seemed quite a while prior. Different fields have been come to by AR innovation yet all in all, there are six sorts of uses, namely close to home information framework, industrial and military applications, restorative applications, entertainment, office applications, instruction and training [1-8]. Yet, still, it discovered a few troubles to achieve end client in this present reality. The invention of a handheld gadget, for example, a cell phone and PC tablet, which had been enormously utilized by individuals as of late truly assisted AR innovation with reaching this present reality application. The study found that the published papers about handheld VR have turned out to be progressively prominent these days. In the training field, Markus et al had built up an AR edutainment content for Sungsan Elementary School (SES) in Busan - South Korea [9]. The work name is EDU tangram and it combined traditional Tangram edutainment device with AR innovation. This work was additionally finalized using handheld gadgets, iPad2. An AR Tangram edutainment was then installed on 30 iPads had a place with SES and they 
utilized it normally during their group session. In the entertainment field, these days there are a few incredible AR projects discharged. In any event there are three major names in the entertainment industry exploring AR innovation for their movement. Transformer makes an AR application for iPad gadget to help their motion picture in the theater. This year Marvel Corp. additionally releases an AR application for a standout amongst their most well-known legend characters, Iron Man. What's more, in conclusion, to help the business arrival of the most up to date Spiderman motion picture in the theater, they additionally make a VR application for the handheld gadget. Together with regular advancement media, for example, publication and site, this handheld $\mathrm{AR}$ application turns into an integrated and innovative marketing device.

\section{3D GAME DESCRIPTION}

War Simulation Multiplayer system through Virtual Reality is the game that we will be building by also taking into references to the other simulations systems around the world. Our main objective is to create a simulation system that will be used by the Army for equipping themselves with various knowledge where in reality will take a lot of resources to accomplish this mere feat. We also wanted to create the simulation in such a way that the simulation can be built with a very minimum rate while also providing the services to use and gain future combat experience just like the usual training regime would proceed. The team and I sat together and researched our way to obtain the very specific objectives that will be used in establishing this simulation. Firstly, we want to create a simulation where multiple users can connect to one master server which will be handled by the commander and from there the various members up to at least a very specific number can join and complete the training workout. This allows the master to have enough flexibility in establishing the various resources and also create a stable environment while also establishing a place where the trainees can their work. Secondly, we wanted to create an offline simulation system inbuilt with the war simulation in order to give a particular user to practice their skills against certain enemy AI. They can be tested in various fields by doing that and a report can be generated referring to the various accomplishments or the areas of improvement that the particular trainee has to work around. In order to provide a fun aspect to the game we added an inventory system which will be able to access the current weapons and be used in game. As of now, there are two types of weapons that can be used they include a Pistol an AK-47 and an M4A1.

Virtual Reality Implication:

Virtual Reality (VR) is an interactive PC produced experience taking spot within a reenacted situation. It incorporates mainly sound-related and visual input, yet may likewise permit other sorts of tangible criticism like haptic. This vivid condition can be like this present reality or it tends to be fantastical. Augmented Reality frameworks may likewise be viewed as a type of VR that layers virtual information over a live camera feed into a headset or through a Cell phone or tablet gadget giving the client the capacity to see three-dimensional pictures. Current VR innovation most normally uses computer generated Reality headsets or multi-projected conditions, at times in combination with physical situations or props, to produce realistic pictures, sounds and other vibes that reproduce a client's physical nearness in a virtual or imaginary condition. An individual using augmented reality gear is ready to "glance around" the fake world, move around in it, and interact with virtual highlights or things. The impact is generally made by VR headsets consisting of a head-mounted display with a little screen before the eyes, yet can likewise be made through uncommonly planned rooms with different enormous screens. VR frameworks that include transmission of vibrations and other sensations to the client through a game controller or other gadgets are known as haptic frameworks. This material information is commonly known as power input and training applications.

\section{Design ANd DeVelopment Process:}

The design of War simulation combines the effects of a normal shooter game combining with a mix of virtual reality and creating an experience where the trainees can use it to train.The specialist utilized the Unity3D game motor is to assemble this game. Unity3D game motor right presently has been broadly utilized by game Designer. There are huge amounts of preferences offer by Unity3D, for example, multi-stage gadget probability (iOS, Android, Xbox, Nintendo Wii, PC), graphical focal points, basic UI, and others. Unity3D is bolstered by 3 basic programming dialects, for example, Java, c\#, and boo. Unity3D additionally has a solid network to help every part here and it turns into another bit of leeway for the different ability scope of client. We also used Blender software in order to design some of the characters that will be used in the game. Blender is a very useful tool that will be used for creating and designing character Terrains weapons and much more which will help us to give the trainees a better experience which shown in figure 1.This is the in-game architecture that will be used to create the game. There are three modules that are in being used.

Online Simulation
Offline Simulation 


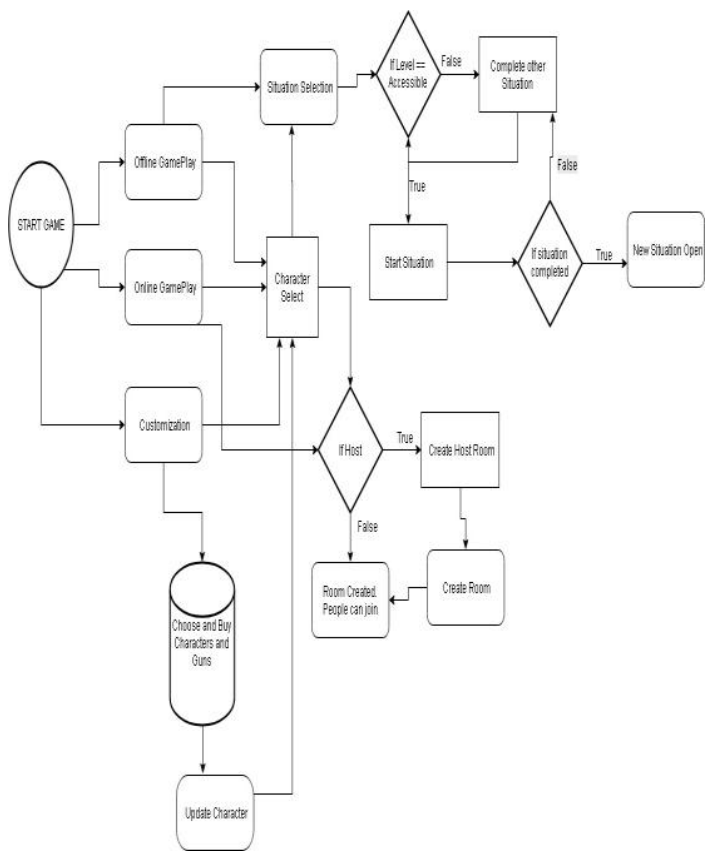

Fig 1- System Architecture

\section{DESIGN AND DEVELOPMENT OF ONLINE SimUlation}

The online simulation here refers to the multiplayer system that will be in use while playing the simulation. This allows multiple users to be in sync and connected with multiple users which will also be monitored by one master server or master Commando. In order to use the online simulation, they should be connected to the Internet and from then there will be a fixed number of users that can participate for the current time in the game. The user first has to start the game and has to select the online game option that will be available in the main menu system. Once the button is being clicked you will be scanned via the available networks that can be connected in a certain range and from there on they will be connected to the nearest network or if possible a shared network space so that the user can connect and access to the simulation. Each user has to connect from their own specific devices and once connected there will be multiple users in one particular game. The design and development process that works together to create the online simulation goes hand in hand the Technical and the design part. The Simulation uses an APK which is known as the Photon Unity Networking or PUN. This particular API allows the developer to make use of this and create a game where you can use the multiplayer and the LAN features. Photon is a real-time multiplayer game development framework that is fast, lean and flexible. Photon consists of a server and multiple client SDKs for major platforms. Photon Unity Network (PUN) is our is our take on a Unity-specific, high-level solution: Matchmaking, easy to use callbacks, components to synchronize Game Objects, Remote Procedure Calls (RPCs) and similar features provide a great start. Beyond that is a solid, extensive API for more advanced control.This plugin is compatible with the managed Photon Cloud service, which runs Photon Servers for you. A setup window registers you (for free) in less than a minute.

Most notable features:

Dead-easy API
A server is available as hosted service.

Load-balanced! Scales across servers

An outstanding performance of the Photon Server

Dedicated servers. No NAT punch-through needed

Offline mode: reuse your multiplayer code in single player game modes.

By combining the elements and the architecture from the photon Unity Network and our own architecture we design a more dynamic multiplayer experience that will be used for the online simulation process.

\section{DESIGN AND DEVELOPMENT OF OFFLINE SIMULATION}

The module offline simulation here refers to the single user gameplay that will be used by only a particular system to access the simulation. In the offline simulation to work, you are not needed to connect the system to the internet instead you can make use of the time and play the game offline that will contain the AI enemies which will help you test out the accomplishments and also the areas of improvement. This will help the users to plan accordingly and play this game. There are a fixed number of missions that can be repeated almost any time and can be played or accessed from anywhere. There is an inventory system that the trainees can access they can manage various weapons that will be suitable for the particular mission. The design and development process involving the creation of a simulation system that can be worked offline is a little less complex when compared to the Online simulation system. We will be making use of A star algorithm in order to set the base for creating an Enemy AI which will be able to detect walk and shoot the Trainee whenever it comes under any contact. The enemy will be having at least basic Artificial Intelligence so that it can work around its surroundings and pose some challenge to the trainee who is working for the improvement of their skills. This is just one of the aspects of the offline simulation system. Another aspect is creating different situation missions which will be pre-built and cannot be replaced. These sets of situations will determine how one trainee is being performed at a particular time. Figure 2 and 3 shown the design models of objects. The calculation keeps up two sets:

- The investigated sets which contain the quantity of states previously investigated. At first vacant.

- The boondocks set which contains the quantity of states going to be investigated. At first contains the underlying state.

The calculation will dependably pick the state from the boondocks with the most reduced aggregate of the present expense and heuristic. This state will at that point be expelled from the outskirts and added to the investigated set. It will at that point grow the state and add the new states to the boondocks. On the off chance that one of the new states is in the investigated set, it will be

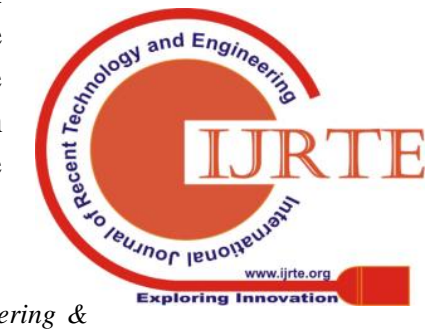

Blue Eyes Intelligence Engineering \& Sciences Publication 
disregarded and on the off chance that one of the new states is in the boondocks with a lower total of current expense and heuristic, it will supplant the current state in the outskirts.

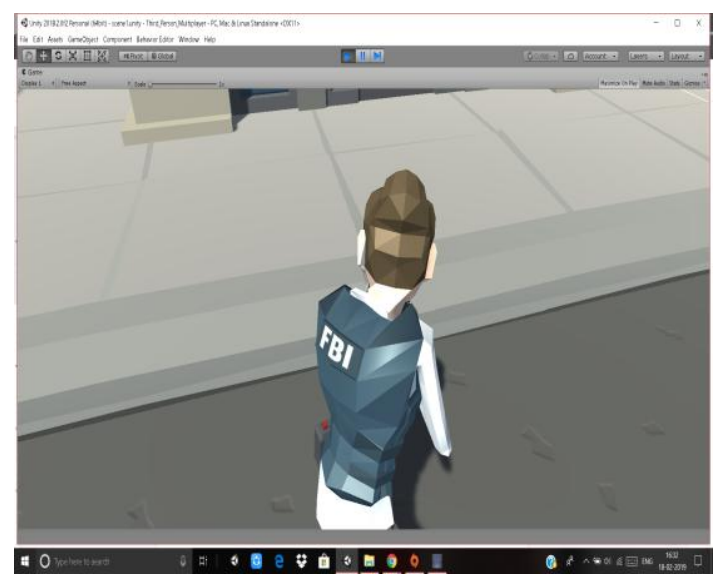

Fig 2 Back view of the character with an AK-47 and an aiming mechanism

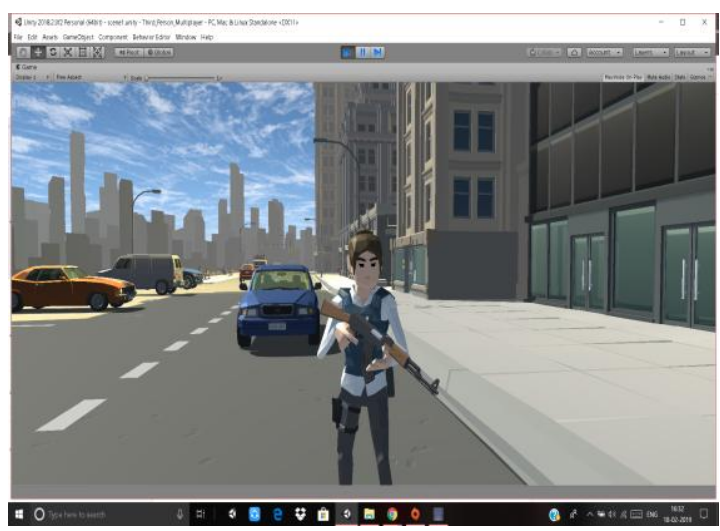

Fig 3. Character holding an AK-47 Assault Rifle

\section{CONCLUSION:}

In this work, we researched the potential development of creating a Simulation system that will be used in training the military by using the medium of Virtual Reality. By doing research we understood the technical requirements and also the design requirements that are to be needed while creating this simulation. By identifying the requirements that are needed to build the simulation we started looking at the different algorithms and also various coding styles that will be required in creating this simulation. We identified that the coding language that will be used here is $\mathrm{C \#}$. But there are also numerous coding languages that can be used in order to meet the specific Coding technicalities. While conducting the research we kept in mind the feasibility requirements and also understood the basic objective that will be used in creating this simulation. By looking at the references of the simulations around the world we like to update the simulation by optimizing it's potential growth while also keeping in mind that it is much more feasible to obtain and maintain. We were able to obtain the API that will be used here in establishing a Network architecture that will help us in creating the online connectivity settings that will be used in establishing networks where multiple people can work on particular master server and use it in such a way that maximum results can be obtained. As we move forward, the researcher hope this research can be a milestone and will inspire further research of the respected field. This will not only help in providing the latest technologies that are needed but also increase the future chances of providing various projects where Virtual Reality, Augmented Reality Serious gaming can be used.

\section{REFERENCES}

1. D.W.F. Van Krevelen, R. Poelman. A Survey of Augmented Reality Technologies, Applications and Limitations. In The International Journal of Virtual Reality, 2010, 9(2), pp1-20.

2. F. Zhou, H.B.L. Duh, M. Billinghurst. Trends in Augmented reality Tracking, Interaction, and Display: A Review of Ten Years of ISMAR In International Symposium on Mixed and Augmented Reality (ISMAR'08)

3. J. Carmigniani, B. Furht. Augmented Reality: An Overview. In Handbook of Augmented Reality. ISBN: 978-1-4614- 0063-9. Springer Science, London

4. M. Billinghurst. Augmented Reality in Education. New Horizons for Learning. Retrieved from: http://www.it.civil.aau.dk/it/education /reports/ar_edu.pdf.2002.

5. M. Billinghurst, A. Henrysson. Research Direction in Handheld AR. In The International Journal of Virtual Reality, 2006, 5(2), pp51-58

6. M. Haller, M. Billinghurst, B. Thomas. Augmented Reality: Interfaces and Design. ISBN: 1-59904-066-2. Idea Group Publishing

7. P. Milgram, F.Kishino. A Taxonomy of Mixed Reality Visual Displays. In IEICE Transactions on Information Systems, VolE77D, No. 12, December 1994.

8. R.T. Azuma. A Survey of Augmented Reality. In Presence:Teleoperators and Virtual Environment, 6:4, 355-385, August 1997.

9. S. Markus, F.Y. Wang, B.G. Lee. Development of Edutainment Content for Elementary School Using Mobile Augmented Reality. Proceeding of ICCRD'12 (Chengdu, China, May 5-6, 2012).

10. Dr. AntoBennet, M ,Resmi R. Nair, Mahalakshmi V,Janakiraman G "Performance and Analysis of Ground-Glass Pattern Detection in Lung Disease based on High-Resolution Computed Tomography",Indian Journal of Science and Technology, Volume09 (Issue02):01-07, January 2016

11. Dr. AntoBennet, M , Sankaranarayanan S, Ashokram S ,Dinesh Kumar T R,"Testing of Error Containment Capability in can Network", International Journal of Applied Engineering Research, Volume 9, Number 19 (2014) pp. 6045-6054.

12. Dr. AntoBennet, M, Sankar Babu G, Natarajan S, "Reverse Room Techniques for Irreversible Data Hiding ", Journal of Chemical and Pharmaceutical Sciences 08(03): 469-475, September 2015

13. Dr. AntoBennet, M , Sankaranarayanan S, Sankar Babu G, “ Performance \& Analysis of Effective Iris Recognition System Using Independent Component Analysis ", Journal of Chemical and Pharmaceutical Sciences 08(03): 571-576, August 2015.

14. Dr. AntoBennet, M, Suresh R, Mohamed Sulaiman S, "Performance \&analysis of automated removal of head movement artifacts in EEG using brain computer interface", Journal of Chemical and Pharmaceutical Research 07(08): 291-299, August 2015.

15. Dr. AntoBennet, M “A Novel Effective Refined Histogram For Supervised Texure Classification", International Journal of Computer \& Modern Technology, Issue 01 Volume02,pp 67-73, June 2015.

16. Dr. AntoBennet, M, Srinath R,Raisha Banu A,"Development of Deblocking Architectures for block artifact reduction in videos", International Journal of Applied Engineering Research, Volume 10, Number 09 (2015) pp. 6985-6991, April 2015.

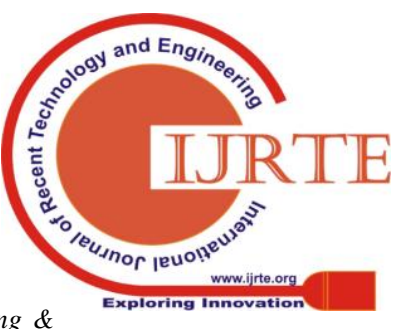

